Artículo

\title{
Calidad de la fresa afectada por la relación nitrato:amonio en la solución nutritiva
}

\author{
Gabriel Lucas de Jesus ${ }^{1 \S}$ \\ Volnei Pauletti ${ }^{2}$ \\ Maria Aparecida Cassilha zawadneak ${ }^{3}$ \\ Francine Lorena Cuquel ${ }^{1}$
}

${ }^{1}$ Departamento de Ciencias de los Cultivos-Universidad Federal de Paraná, Brasil. R. dos Funcionários 1540, Curitiba, Brasil. CP. 80035-050. (francine@ufpr.br). ${ }^{2}$ Departamento de Ingeniería de Suelos y Agricultura-Universidad Federal de Paraná, Brasil. R. dos Funcionários 1540, Curitiba, Brasil. CP. 80035050. (vpauletti@ufpr.br). ${ }^{3}$ Departamento de Patología Básica-Universidad Federal de Paraná, Brasil. Av. Cel. Francisco H. dos Santos 100, Curitiba, Brasil. CP. 81531-980. (mazawa@ufpr.br).

Autor para correspondencia: gabriel.lucas@ufpr.br.

\section{Resumen}

La producción de fresa (Fragaria x ananassa Duch.) mediante el uso del sistema de fertirrigación semihidropónico es una tecnología cada vez más adoptada. Uno de los principales desafíos de este sistema es el manejo nutricional del nitrato $\left(\mathrm{NO}_{3}^{-}\right)$y el amonio $\left(\mathrm{NH}_{4}{ }^{+}\right)$, que pueden afectar el desarrollo y la calidad de los frutos. Esta investigación tuvo como objetivo determinar la mejor relación $\mathrm{NO}_{3}{ }^{-} \mathrm{NH}_{4}{ }^{+}$para una mejor calidad de frutos de fresa cultivados en un sistema semihidropónico. Los corredores de fresa 'San Andreas' se plantaron en sustrato orgánico compuesto por cáscara de arroz carbonizada y corteza de pino. Las proporciones de $\mathrm{NO}_{3}{ }^{-}: \mathrm{NH}_{4}{ }^{+}$ evaluadas en fertirrigación fueron 100: 0; 75:25; 50:50; 25:75 y 0:100. El contenido de nitrógeno de las hojas y la pérdida de masa seca aérea mostraron un aumento proporcional concomitantemente con un aumento de $\mathrm{NH}_{4}{ }^{+}$en la solución. El área foliar y el contenido de clorofila aumentaron hasta $29 \%{\mathrm{de} \mathrm{NH}_{4}}^{+}$en la solución nutritiva, mientras que concentraciones más altas afectaron negativamente estas características. Las características químicas de las frutas no se vieron afectadas por la relación $\mathrm{NO}_{3}{ }^{-} \mathrm{NH}_{4}{ }^{+}$, pero la firmeza de la pulpa disminuyó a medida que aumentaba el $\mathrm{NH}_{4}{ }^{+}$en la solución nutritiva. Los resultados obtenidos mostraron que los frutos de fresa con mejor desarrollo y calidad se obtuvieron aplicando $\mathrm{NO}_{3}{ }^{-} \mathrm{NH}_{4}{ }^{+}$en solución de fertirrigación en la proporción de 71:29.

Palabras clave: Fragaria x ananassa Duch., cultivo sin suelo, nutrición de amonio, 'San Andreas'.

Recibido: marzo de 2021

Aceptado: junio de 2021 


\section{Introducción}

El cultivo de fresas en el sistema semihidropónico, conocido popularmente como cultivo sin suelo, es una práctica aplicada en varias regiones del planeta (Recamales et al., 2007; Neri et al., 2012; Rampazzo, 2016). En lugar de suelo, utiliza una amplia gama de materias primas para producir los sustratos, como la perlita, la vermiculita, la turba, la corteza de pino, la cáscara de arroz carbonizada y la fibra de coco (Lieten et al., 2004; Jarosz y Konopinska, 2010; Marques, 2016). Los sustratos utilizados en el sistema semihidropónico suelen tener buenas características físicas y químicas y tienen una buena biodisponibilidad de nutrientes para el desarrollo de las plantas (Abad et al., 2005; Ilha, 2013). Además, están libres de plagas y enfermedades, proporcionan un uso más racional del agua y los nutrientes y son más ergonómicos para los trabajos (Rampazzo, 2016).

Uno de los puntos más críticos en el sistema semihidropónico de la fresa es el manejo del nitrógeno mediante fertirrigación (Othman et al., 2019). La disponibilidad de nitrógeno afecta la productividad de las fresas y la calidad de los frutos (Nestby et al., 2004; Jarosz y Konopiska, 2010). Ambas formas de nitrógeno, nitrato y sales de amonio, son absorbidas y metabolizadas por las plantas (Roosta et al., 2009) y desempeñan funciones metabólicas importantes, como el crecimiento, el desarrollo, la producción y afectan la apariencia, durabilidad y sabor de los frutos (Tabatabaei et al., 2008; Krüger et al., 2012; Samec et al., 2016).

A pesar de esto, hay poca información sobre la concentración de nitratos y amonio en soluciones

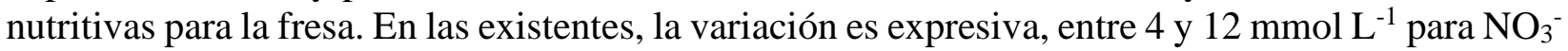
y 0.2 y $2.5 \mathrm{mmol} \mathrm{L}^{-1}$ para $\mathrm{NH}_{4}{ }^{+}$(Hennion y Veschambre, 1997; Paranjpe et al., 2003; Furlani y Fernández, 2004). Esta investigación tuvo como objetivo determinar la mejor proporción de $\mathrm{NO}_{3}{ }^{-}$ $: \mathrm{NH}_{4}{ }^{+}$para la producción de fresas mediante el uso del sistema de fertirrigación semihidropónico.

\section{Materiales y métodos}

Esta prueba se realizó en Curitiba, estado de Paraná, Brasil (2524’38.5” S; 49 14' 57. 5" O). Cada corredor de fresa 'San Andreas', un cultivar neutro al fotoperiodo, fue plantado en macetas de polipropileno negro de 8 litros el 09/07/18, llenas con sustrato orgánico (Cuadro 1) compuesto por cáscara de arroz y corteza de pino.

Cuadro 1. Características del sustrato orgánico utilizado para el cultivo de corredores de fresa 'San Andreas' en un sistema semihidropónico.

\begin{tabular}{|c|c|c|c|c|c|c|c|}
\hline \multirow{2}{*}{$\mathrm{pH} \mathrm{CaCl} 2$} & \multirow{2}{*}{$\mathrm{DD}\left(\mathrm{kg} \mathrm{m}^{-3}\right)$} & \multirow{2}{*}{$\mathrm{EC}\left(\mathrm{mS} \mathrm{cm}^{-1}\right)$} & \multirow{2}{*}{$C\left(\mathrm{~g} \mathrm{dm}^{-3}\right)$} & $\mathrm{TP}$ & RAW & $\mathrm{BS}$ & $\mathrm{AW}$ \\
\hline & & & & \multicolumn{4}{|c|}{$(\%)$} \\
\hline 5.65 & 266.78 & 399 & 151.3 & 84.4 & 7.96 & 1.59 & 9.55 \\
\hline
\end{tabular}

$\mathrm{dd}=$ densidad seca; $\mathrm{tp}=$ porosidad total; $\mathrm{raw}=$ agua fácilmente disponible; $\mathrm{bs}=$ solución amortiguadora; aw= agua disponible.

El diseño experimental aplicado fue completamente aleatorizado con cinco repeticiones por tratamiento, y cada repetición con tres macetas. Los tratamientos consistieron en proporciones de nitrato y amonio $\left(\mathrm{NO}_{3}{ }^{-} \mathrm{NH}_{4}{ }^{+}\right)$en la solución nutritiva de 100:0; 75:25; 50:50; 25:75 y 0:100\%, con $150 \mathrm{mg} \mathrm{L}^{-1}$ de concentración de nitrógeno aplicada en todas las macetas, con base y modificada por Furlani y Fernandes Júnior (2004) (Cuadro 2). 
Cuadro 2. Macronutrientes y concentración de cloro en la solución nutricional utilizada, de acuerdo con cada proporción de nitrato: amonio.

\begin{tabular}{ccccc}
\hline & $\mathrm{NO}_{3}{ }^{-}$ & $\mathrm{NH}_{4}{ }^{+}$ & $\mathrm{SO}_{4}{ }^{2-}$ & $\mathrm{Cl}^{-}$ \\
\cline { 2 - 5 } & \multicolumn{3}{c}{$\left(\mathrm{mg} \mathrm{L}^{-1}\right)$} \\
\hline $100-0$ & 150 & 0 & 48.9 & 131 \\
$75-25$ & 112.5 & 37.5 & 90 & 249.8 \\
$50-50$ & 75 & 75 & 112.5 & 423.1 \\
$25-75$ & 37.5 & 112.5 & 153.6 & 544.8 \\
$0-100$ & 0 & 150 & 194.6 & 666.1 \\
\hline
\end{tabular}

Otros nutrientes se aplicaron en las mismas concentraciones en todas las macetas: $170 \mathrm{mg} \mathrm{L}^{-1} \mathrm{de}$ $\mathrm{Ca}, 200 \mathrm{mg} \mathrm{L}^{-1}$ de $\mathrm{K}, 40 \mathrm{mg} \mathrm{L}^{-1}$ de $\mathrm{HPO}_{4}{ }^{-}, 40 \mathrm{mg} \mathrm{L}^{-1}$ de $\mathrm{Mg}, 32 \mu \mathrm{mol} \mathrm{L}{ }^{-1}$ de Fe, $10 \mu \mathrm{mol} \mathrm{L}^{-1}$ de $\mathrm{Mn}$, $30 \mu \mathrm{mol} \mathrm{L}{ }^{-1}$ de $\mathrm{B}, 3.1 \mu \mathrm{mol} \mathrm{L}^{-1}$ de $\mathrm{Zn}, 1.3 \mu \mathrm{mol} \mathrm{L}^{-1}$ de $\mathrm{Cu}$ y $0.17 \mu \mathrm{mol} \mathrm{L}^{-1}$ de Mo. Para la formulación de la solución nutritiva se utilizaron las siguientes sales: $\mathrm{Ca}\left(\mathrm{NO}_{3}\right)_{2}, \mathrm{KNO}_{3}, \mathrm{KH}_{2} \mathrm{PO}_{4}$, $\mathrm{MgSO}_{4}, \mathrm{NH}_{4} \mathrm{H}_{2} \mathrm{PO}_{4}, \mathrm{CaCl}_{2}, \mathrm{KCl},\left(\mathrm{NH}_{4}\right)_{2} \mathrm{SO}_{4}, \mathrm{H}_{3} \mathrm{BO}_{3}, \mathrm{CuSO}_{4}, 5 \mathrm{H}_{2} \mathrm{O}, \mathrm{MnSO}_{4} \mathrm{H}_{2} \mathrm{O}, \mathrm{ZnSO}_{4} 7 \mathrm{H}_{2} \mathrm{O}$, $\mathrm{Na}_{2} \mathrm{MoO}_{4} 2 \mathrm{H}_{2} \mathrm{O}, \mathrm{Fe}^{-} \mathrm{EDDHA}(6 \%)$.

Se aplicó fertirrigación en cada maceta una vez al día, para mantener la capacidad de retención de agua del sustrato en $60 \%$, de esta manera se aplicaron aproximadamente $500 \mathrm{ml}$ diarios por maceta. La conductividad eléctrica (EC) en la solución nutricional se mantuvo entre 1300 y 1 $800 \mathrm{mS} \mathrm{cm}^{-1}$ y el pH en $6 \pm 0.2$.

Las evaluaciones de las hojas se realizaron a 60 y 120 días después de la siembra (Dap), correspondientes a las etapas vegetativa y productiva, respectivamente (Nepar, 2019). El área foliar se obtuvo midiendo la longitud y anchura de los foliolos de la cuarta hoja recién desarrollada de la planta con un calibrador digital y utilizando la ecuación $\mathrm{La}=(\pi / 4)[(\mathrm{L}+\mathrm{W}) / 2]^{2}$ (Pires, 1999). Donde: $\mathrm{La}=$ área foliar; $\mathrm{L}=$ longitud y $\mathrm{W}=$ anchura. A partir de los mismos foliolos se recogió una muestra de tejido de $0.17 \mathrm{~g}$ para evaluar la clorofila a, b y las hojas totales (Porra, 2002).

El material fue macerado con $10 \mathrm{ml}$ de acetona al $80 \%$ y centrifugado a $12000 \mathrm{rpm}$ durante 10 minutos y se realizó una lectura del espectrofotómetro a longitudes de onda de 645 y $663 \mathrm{~nm}$. El resto de la cuarta hoja recogida se utilizó para el análisis nutricional ( $\mathrm{S}, \mathrm{Ca}, \mathrm{K}, \mathrm{Mg}, \mathrm{P}, \mathrm{Fe}$ y $\mathrm{Zn}$ ) mediante digestión húmeda con ácido nítrico $\left(\mathrm{HNO}_{3}\right)$ y peróxido de hidrógeno $\left(\mathrm{H}_{2} \mathrm{O}_{2}\right)$, y las lecturas se hicieron con la espectroscopía de emisión óptica de plasma acoplado inductivamente (ICP-OES) (Varian 720-ESTM). El N total fue analizado por el método de combustión de Dumas en un Analizador Elemental (Carmo et al., 2000).

Todas las hojas senescentes se recogieron durante el crecimiento de las plantas y se secaron a 65 ${ }^{\circ} \mathrm{C}$ hasta alcanzar una masa constante. Al final del ensayo, todas las hojas que quedaban también se secaron a $65{ }^{\circ} \mathrm{C}$ hasta alcanzar una masa constante. El peso seco por planta se obtuvo añadiendo el peso seco de senescencia al peso seco final de las hojas.

Se analizaron las características físicas y químicas de los frutos en diez frutos cosechados por réplica, en tres fechas de cosecha (20/12/2017; 20/01/2018; 20/02/2018). Se eligieron aquellos con al menos 3/4 de epidermis rojiza, peso mínimo de $6 \mathrm{~g}$ y sin lesiones, enfermedades $\mathrm{o}$ 
deformaciones. En primer lugar, la firmeza de la pulpa se determinó utilizando un analizador de textura (Brookfield CT3 ${ }^{\mathrm{TM}}$ ), con punta de $2 \mathrm{~mm}$, penetración de $5 \mathrm{~mm}$ y velocidad de penetración de $5 \mathrm{~mm} \mathrm{~s}^{-1}$. Posteriormente, se extrajo el jugo de fresa, se midió el contenido de sólidos solubles usando un refractómetro. La solución del jugo se diluyó en agua al $10 \%$ y la titulación se realizó con $0.1 \mathrm{M}$ de solución de hidróxido de sodio $(\mathrm{NaOH})$ bajo agitación constante hasta un pH de 8.2.

Se utilizó la prueba de Bartlett para verificar la homogeneidad de las varianzas $(p>0.05)$ de los datos, seguida de Anova. En caso de variación significativa, se utilizó el análisis de regresión para verificar los efectos de las proporciones de $\mathrm{NO}_{3}{ }^{-}: \mathrm{NH}_{4}{ }^{+}$. Para el análisis estadístico se utilizó el software Assistat 7.7 (Silva, 2002).

\section{Resultados y discusión}

Las proporciones de $\mathrm{NO}_{3}{ }^{-}: \mathrm{NH}_{4}{ }^{+}$no afectaron la mayoría de los contenidos de nutrientes de las hojas $\left(\mathrm{S}=1.19 \mathrm{~g} \mathrm{~kg}^{-1} ; \mathrm{Ca}=10.64 \mathrm{~g} \mathrm{~kg}^{-1} ; \mathrm{K}=18.13 \mathrm{~g} \mathrm{~kg}^{-1} ; \mathrm{Mg}=3.75 \mathrm{~g} \mathrm{~kg}^{-1} ; \mathrm{P}=2.89 \mathrm{~g} \mathrm{~kg}^{-1} ; \mathrm{Fe}=69.42 \mathrm{mg}\right.$ $\mathrm{kg}^{-1} ; \mathrm{Zn}=23.38 \mathrm{mg} \mathrm{kg}^{-1}$ ). Estos niveles de nutrientes son similares al nivel foliar en hojas de fresa reportado por otros autores (CQFS RS/SC, 2004; Nepar, 2019). Se esperaba que el nivel más alto de $\mathrm{NH}_{4}{ }^{+}$en la solución nutritiva se afectaran los niveles de $\mathrm{Ca}, \mathrm{K}$ y $\mathrm{Mg}$; a través, de la competencia entre iones de la misma valencia, ya sea por un canal proteico o por unión a una proteína transportadora en la membrana celular (White, 2012), pero eso no ocurrió en esta investigación.

Tampoco se apreció un cambio en el pH en la rizosfera por la absorción de $\mathrm{NH}_{4}{ }^{+}$(Hawkesford et al. (2012), lo que tiene un efecto directo sobre la biodisponibilidad de nutrientes (Waller y Wilson, 1984). El pH del sustrato cambió poco durante este ensayo (5.65 antes de la siembra -5.27 en 0$100 \%$ de $\mathrm{NO}_{3}{ }^{-}: \mathrm{NH}_{4}{ }^{+}$), debido a su composición orgánica. La composición del sustrato juega un papel importante dado que Choi et al. (2011) encontró una disminución del pH del sustrato, de un $\mathrm{pH}$ de 7 con el uso de solución nutritiva sin $\mathrm{NH}_{4}{ }^{+}$a un $\mathrm{pH}$ de 5.8 con $\mathrm{NH}_{4}{ }^{+}$y también una

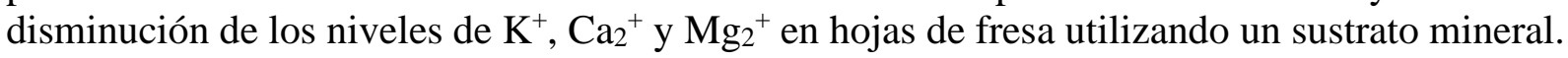

De los nutrientes evaluados en la hoja, sólo el nitrógeno total $(\mathrm{N})$ se vio afectado por las diferentes proporciones de $\mathrm{NO}_{3}{ }^{-} \mathrm{NH}_{4}{ }^{+}$de las soluciones nutritivas. El contenido fue de $20.64 \mathrm{~g} \mathrm{~kg}^{-1}$, cuando el $100 \%$ del nitrógeno aplicado estuvo como $\mathrm{NH}_{4}{ }^{+}$, que presenta un aumento de aproximadamente $14 \%$ de $\mathrm{N}$ en relación con la ausencia de $\mathrm{NH}_{4}{ }^{+}$(Figura 1). Un resultado similar fue obtenido por Tabatabaei et al. (2008), con la aplicación de la dosis máxima de $75 \%$ de $\mathrm{NH}_{4}{ }^{+}$en la variedad de fresa 'Selva' se encontró un aumento de alrededor de $16.8 \%$ en el contenido foliar de $\mathrm{N}$ y por Choi et al. (2011), con un aumento de $14.2 \%$ de $\mathrm{N}$ al utilizar $100 \%$ de $\mathrm{NH}_{4}{ }^{+}$en la solución nutritiva.

La mayoría de las plantas absorben las dos formas de nitrógeno que están mediadas por proteínas de transporte específicas (Roosta et al., 2009). Las formas de nitrógeno pueden ser metabolizadas en la misma célula de la raíz donde fue absorbida o transladada sin cambios a la parte aérea de la planta. El $\mathrm{NH}_{4}{ }^{+}$se asimila en las células de la raíz y su forma reducida se lleva a las hojas, mientras que la mayor parte de $\mathrm{NO}_{3}{ }^{-}$se lleva a las hojas y luego se reduce para ser asimilado en aminoácidos. El requerimiento energético para la asimilación $\mathrm{NH}_{4}{ }^{+}$es menor al requerido para la asimilación de $\mathrm{NO}_{3}{ }^{-}$ya que el primero no necesita ser reducido para su incorporación en aminoácidos (Bloom et al., 1992; Hawkesford et al., 2012; Taiz y Zeiger, 2017). Posiblemente, el $\mathrm{NH}_{4}{ }^{+}$asimilado en las raíces y transportado en forma de aminoácidos terminó acumulándose en las hojas y aumentando los niveles de $\mathrm{N}$ en estos tejidos (Majerowicz et al., 2000). 


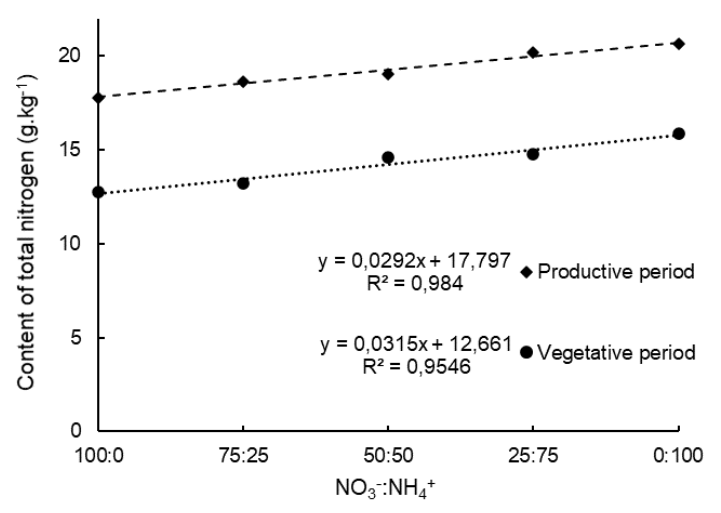

Figura 1. Contenido de nitrógeno total en hojas de fresa del cultivar 'San Andreas' con proporciones de $\mathrm{NO}_{3}{ }^{-}: \mathrm{NH}_{4}{ }^{+}$en la solución nutritiva, en período vegetativo (60 días después de la siembra) y período reproductivo (120 días después de la siembra), en cultivo semihidropónico.

Las altas concentraciones de $\mathrm{NH}_{4}{ }^{+}$en la solución nutritiva causaron necrosis en las hojas (Figura 2), aumentando la masa seca de la parte aérea (Figura 3), indicando un efecto tóxico sobre la planta. Choi et al. (2011) observaron que en altas concentraciones de $\mathrm{NH}_{4}{ }^{+}$en la solución nutritiva, las hojas jóvenes de fresa presentaron un color verde mate, con bordes marchitos y rizados, mientras que las hojas viejas estaban secas y bronceadas. El mayor contenido de $\mathrm{N}$ en la hoja (Figura 1) favoreció la producción de $\mathrm{NH}_{4}{ }^{+}$a través de la fotorrespiración, como la vía metabólica para la producción de este compuesto en plantas (Bittsánszky et al., 2015), dando lugar a la toxicidad.
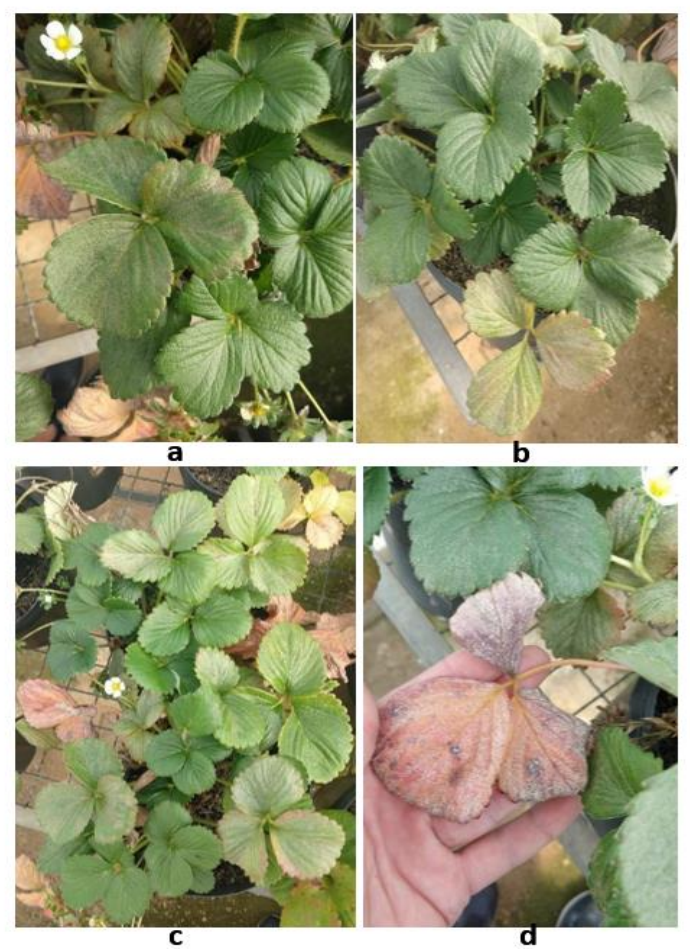

Figura 2. Síntomas relacionados con el efecto tóxico de $\mathrm{NH}_{4}{ }^{+}$en plantas de fresa bajo tratamiento de 0:100\% de $\mathrm{NO}_{3}{ }^{-} \mathrm{NH}_{4}{ }^{+}$en la solución nutritiva, donde: a) inicio de síntomas; b) síntoma agravado; c) vista aérea de la planta; y d) muerte de hojas. 


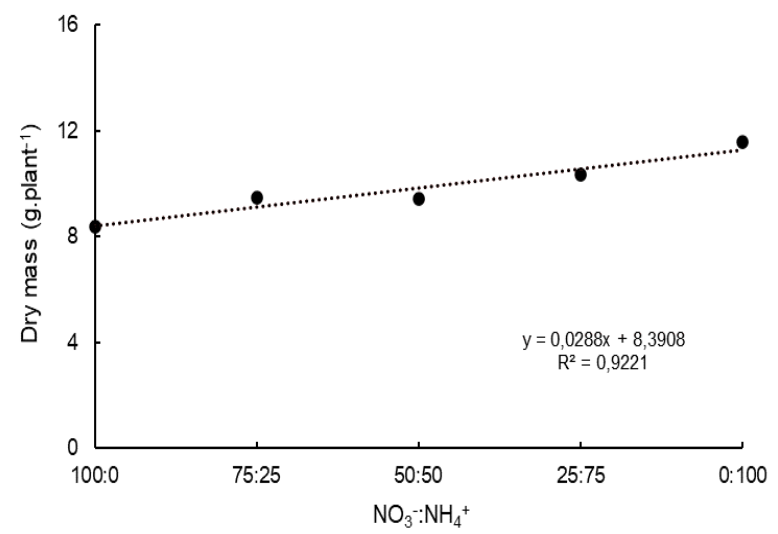

Figura 3. Masa seca del cultivar 'San Andreas' después de 120 días de la plantación, de acuerdo con proporciones de $\mathrm{NO}_{3}{ }^{-}: \mathrm{NH}_{4}{ }^{+}$en la solución nutritiva en cultivo semihidropónico.

La toxicidad ocurre cuando la tasa de asimilación de $\mathrm{NH}_{4}{ }^{+}$en aminoácidos y amidas se vuelve más baja que la tasa de absorción. En las hojas, la acumulación de $\mathrm{NH}_{4}{ }^{+}$causa daño a las estructuras del cloroplasto, bajando el $\mathrm{pH}$ de las células a niveles intolerables. Este hecho disipa los gradientes de protones transmembrana necesarios para el transporte de electrones en la fotosíntesis, lo que resulta en una disminución en la tasa fotosintética (Bittsánszky et al., 2015).

Los niveles de clorofila variaron de manera similar en los períodos de 60 DAP y 120 DAP, bajo las proporciones de $\mathrm{NO}_{3}{ }^{-}: \mathrm{NH}_{4}{ }^{+}$(Figura 4). Los contenidos máximos de clorofila fueron de 0.250 $\mathrm{mg} \mathrm{g}^{-1}$ en las proporciones de $36.2 \%$ de $\mathrm{NH}_{4}{ }^{+}$. Tabatabaei et al. (2008) también observaron un aumento del contenido de clorofila utilizando la proporción de $50 \%$ de $\mathrm{NH}_{4}{ }^{+}$en la solución nutritiva. En el presente estudio, el aumento de la concentración de $\mathrm{NH}_{4}{ }^{+}$por encima de $36 \%$ disminuyó el contenido de clorofila de la hoja, probablemente debido a la fitotoxicidad de $\mathrm{NH}_{4}{ }^{+}$ discutida anteriormente. Los niveles máximos de clorofila se obtuvieron con $\mathrm{NO}_{3}{ }^{-}$y $\mathrm{NH}_{4}{ }^{+}$en la solución de fertirrigación, en la que el protón generado por la asimilación de $\mathrm{NH}_{4}{ }^{+}$puede ser utilizado para reducir el $\mathrm{NO}_{3}{ }^{-}$, facilitando así que las plantas regulen el $\mathrm{pH}$ intracelular cuando se proporcionan ambas formas de nitrógeno (Hawkesford et al., 2012).
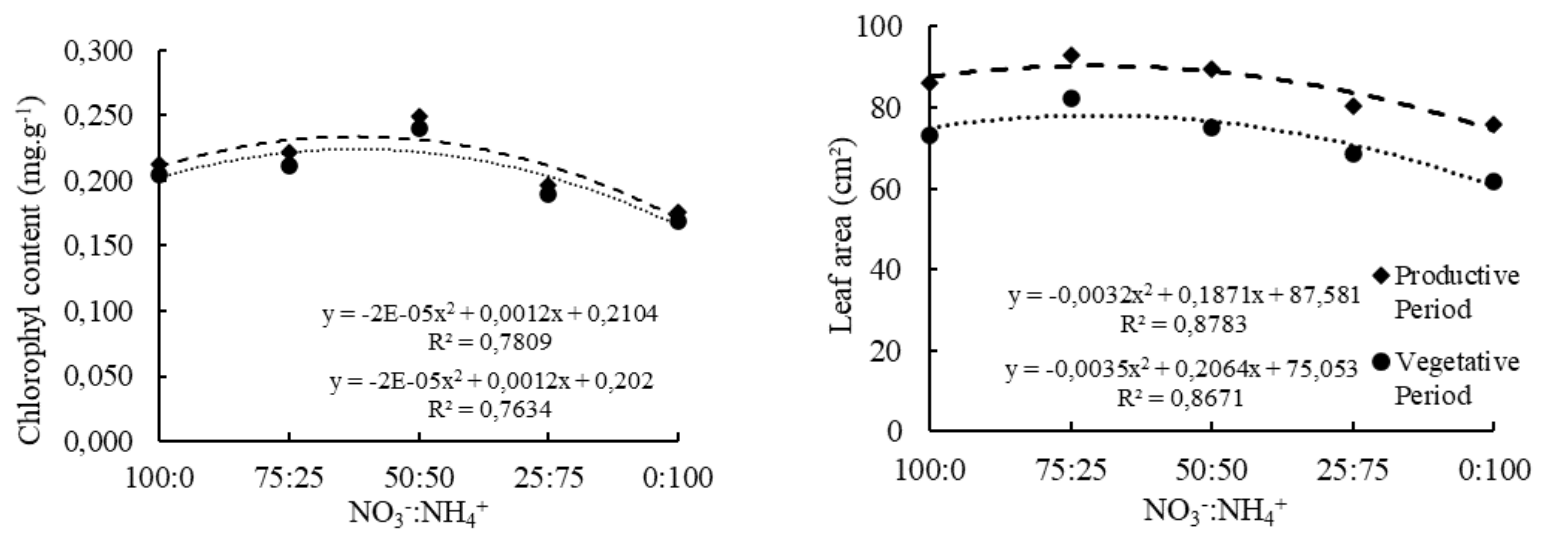

Figura 4. Contenido de clorofila y área foliar de las hojas de fresa 'San Andreas' con las proporciones de $\mathrm{NO}_{3}: \mathrm{NH}_{4}{ }^{+}$en la solución nutritiva, en período vegetativo (60 días después de la siembra) y período reproductivo (120 días después de la siembra) en cultivo semihidropónico. 
La combinación adecuada de $\mathrm{NO}_{3}{ }^{-}: \mathrm{NH}_{4}{ }^{+}$tiene un efecto estimulante en las plantas porque reduce el gasto de energía en el proceso de absorción de nitrógeno, realizado por las células de la raíz y en la asimilación en aminoácidos. Por lo tanto, la célula con su metabolismo regulado y con mayor eficiencia energética puede asimilar el nitrógeno en glutamina y glutamato, así como incorporar otros aminoácidos que juegan un papel importante en la formación de proteínas y moléculas, como la clorofila (Taiz y Zeiger, 2017).

La relación 71:29 de $\mathrm{NO}_{3}{ }^{-} \mathrm{NH}_{4}{ }^{+}$fue la que proporcionó mayor área foliar, tanto en 60 DAP como en 120 DAP, alcanzando $93 \mathrm{~cm}^{2}$ (Figura 4). Estos valores se aproximan a los estudios realizados por Tabatabaei et al. (2008); Choi et al. (2011), en el que la aplicación de 25\% de nitrógeno en forma de $\mathrm{NH}_{4}{ }^{+}$dio lugar a mayores áreas foliares. Fue posible una mayor expansión foliar, ya que la regulación osmótica y energética proporcionada por las dos formas de $\mathrm{N}\left(\mathrm{NO}_{3}{ }^{-}\right.$y $\left.\mathrm{NH}_{4}{ }^{+}\right)$benefició el alargamiento celular y el crecimiento de las plantas (Cao y Li, 2003; Li et al., 2013).

Los resultados del área foliar (Figura 4) muestran la misma tendencia que los contenidos de clorofila (Figura 4), ya que la clorofila es la base para la formación de diversos componentes del aparato fotosintético de la planta, proporcionando un mayor desarrollo foliar. Sin embargo, hubo un menor crecimiento foliar en plantas suplementadas con alta concentración de $\mathrm{NH}_{4}{ }^{+}(75 \%$ y $100 \%$ ), probablemente debido a una mayor demanda de carbohidratos que se canalizan para la asimilación y desintoxicación de la gran cantidad de este catión.

La firmeza de la pulpa fue la única característica fisicoquímica que guía la calidad del fruto afectada por las proporciones de $\mathrm{NO}_{3}{ }^{-}: \mathrm{NH}_{4}{ }^{+}$y disminuyó hasta $25 \%$ con el aumento de los niveles de $\mathrm{NH}_{4}{ }^{+}$ en la solución nutritiva (Figura 5). Es similar a los resultados encontrados por Sokri et al. (2015) en manzanas, donde la mayor concentración de $\mathrm{NH}_{4}{ }^{+}$que fue probada en la solución disminuye la firmeza de la pulpa.

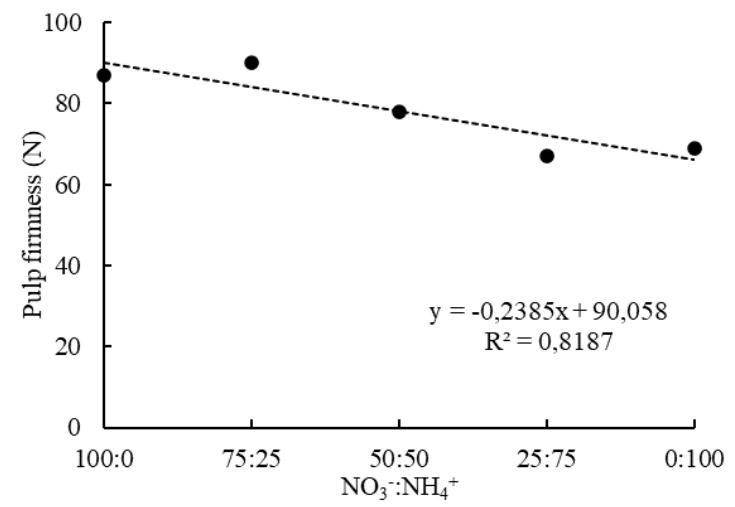

Figura 5. Firmeza de la pulpa del fruto de fresa cultivar 'San Andreas' de acuerdo con proporciones de $\mathrm{NO}_{3}{ }^{-}: \mathrm{NH}_{4}{ }^{+}$en la solución nutritiva en cultivo semihidropónico.

La firmeza de la pulpa está estrechamente relacionada con el $\mathrm{Ca}$, ya que está ligada a ácidos poligalacturónicos como las pectinas. Ellos forman los pectatos de Ca en la laminilla media, cuya función es esencial para la formación de paredes celulares y tejidos vegetales. La degradación del pectato está mediada por la poligalacturonasa, una enzima fuertemente inhibida por altas concentraciones de $\mathrm{Ca}$ (Wehr et al., 2004). Así, cuando se produce una deficiencia de Ca, se 
incrementa la actividad de la poligalacturonasa (Konno et al., 1984). Un síntoma típico de la deficiencia de $\mathrm{Ca}$ es la desintegración de las paredes celulares y la descomposición de los tejidos afectados, lo que causa aflojamiento y pérdida de la firmeza del fruto (Ho y White, 2005). La reducción en la firmeza de la pulpa puede estar relacionada con menor concentración de Ca en los frutos (Alan, 1989). En este experimento, aunque las concentraciones foliares de Ca no se vieron afectadas por $\mathrm{NH}_{4}{ }^{+}$, es probable que las concentraciones de $\mathrm{Ca}$ en el fruto hayan sido afectadas.

Los frutos presentaron valores medios de sólidos solubles totales (TSS) de 8.5, en ${ }^{\circ}$ Brix, acidez total titulable (TTA) en \% de ácido cítrico de 0.97; TSS/TTA (proporción) de 8.9 y pH de 3.5. Según Kader (1999), para que el sabor de fresa sea aceptable, la cantidad mínima de TSS es alrededor de $7{ }^{\circ}$ Brix. Los valores máximos de acidez recomendados se encuentran entre $0.8 \%$ y la relación SS/TA de al menos 8.75. Se esperaba que los niveles de SST disminuyeran y los niveles de TTA aumentaran con concentraciones altas de $\mathrm{NH}_{4}^{+}$en la solución nutritiva.

Esto porque, según Sokri et al. (2015); Zhang et al. (2019), la aplicación de $\mathrm{NH}_{4}{ }^{+}$disminuye la afluencia de K por la planta, debido al antagonismo de estos cationes. La concentración de cationes se reduce con el aumento de la concentración de amonio, que puede conducir a un aumento en la acidez de los frutos. Por otro lado, en semillas de soja y frutos de manzana (Malus domestica Borkh. cv. Fuji), la fertilización con K aumentó el TSS (Tu et al., 2017; Zhang et al., 2017). Aunque los tejidos foliares muestran síntomas de toxicidad de $\mathrm{NH}_{4}{ }^{+}$, en este ensayo, el contenido de $\mathrm{K}$ de la hoja no varió con el aumento de $\mathrm{NH}_{4}{ }^{+}$, manteniendo las características químicas de los frutos.

\section{Conclusiones}

En este trabajo, el daño causado por el exceso de $\mathrm{NH}_{4}{ }^{+}$en la solución nutritiva fue mitigado mediante el uso de un sustrato orgánico en el cultivo de fresas. Teniendo en cuenta lo anterior, los mecanismos exactos de la toxicidad causada por $\mathrm{NH}_{4}{ }^{+}$no son totalmente comprensibles. Se recomiendan estudios futuros para dilucidar mejor cómo afectó el $\mathrm{NH}_{4}{ }^{+}$a la firmeza de la pulpa de fresa. La mejor relación de $\mathrm{NO}_{3}{ }^{-}: \mathrm{NH}_{4}{ }^{+}$para el desarrollo y la calidad de la fresa mediante el uso del sistema de fertiirrigación semihidropónica es de 71:29.

\section{Literatura citada}

Abad, M.; Fornes, F.; Carrión, C.; Noguera, V.; Noguera, P.; Maquieira, A. and Puchades, R. 2005. Physical properties of various coconut coir dusts compared to peat. HortScience. 40(7):2138-2144. Doi: https://doi.org/10.21273/hortsci.40.7.2138.

Alan, R. 1989. The effect of nitrogen nutrition on growth, chemical composition and response of cucumber Cucumis sativius L. to nitrogen forms in solution culture. J. Hortic. Sci. 64(4):467-474. Doi: https://doi.org/10.1080/14620316.1989.11515979.

Bittsánszky, A.; Pilinszkya, K.; Gyulaib, G. and Komives, T. 2015. Overcoming ammonium toxicity. Plant Sci. 231(2):184-190. Doi: 10.1016/j.plantsci.2014.12.005.

Bloom, A. J.; Sukrapanna, S. S. and Warner, R. L. 1992. Root respiration associated with ammonium and nitrate absorption and assimilation by barley. Plant Physiol. 99(4):12941301. Doi: https://doi.org/10.1104/pp.99.4.1294. 
Cao, C. L. and Li, S. X. 2003. Effects of N form on some physiological characteristics and yield of wheat during the vegetative reproductive growth stages. Acta Agr. Singapore. 29(2):258-262.

Carmo, C. A. F. S.; Araujo, W. S.; Bernardi, A. C. C. y Saldanha, M. F. C. 2000. Metodos de análise de tecidos vegetais utilizados na embrapa solos. Río de Janeiro. Embrapa Solos. $41 \mathrm{p}$.

CQFS, RS/SC. 2004. Comissão de química e fertilidade do solo. Manual de adubação e de calagem para os estados do Rio Grande do Sul e de Santa Catarina. 10. (Ed.). Porto Alegre, Sociedade Brasileira De Ciência do Solo. 400 p.

Choi, J. M.; Latigui, A. and Lee, C. W. 2011. Growth and nutrient uptake responses of 'seolhyang' strawberry to various ratios of ammonium to nitrate nitrogen in nutrient solution culture using inert media. Afr. J. Biotechnol. 10(59):12567-12574. Doi:10.5897/ajb11.1104.

Furlani, P. R. and Fernandez, J. F. 2004. Cultivo hidropônico de morango em ambiente protegido. In: simpósio nacional do morango and encontro de pequenas frutas e frutas nativas do mercosul pelotas. Anais pelotas: corrêa antunez, $1^{\mathrm{e}}$ (Ed.). Embrapa. 102-115 p.

Hennion, B. and Veschambre, D. 1997. La fraise: maîtrise de la production. Paris. CTFIL. 299 p.

Ho, L. and White, P. J. A. 2005. Cellular hypothesis for the induction of blossom end rot in tomato fruit. Ann. Bot. 95(4):571-581. Doi: https://doi.org/10.1093/aob/mci065.

Ilha, L. H. 2013. Produção de morango semi-hidropônico. In: seminário brasileiro sobre pequenas frutas. Embrapa Uva e Vinho. 41 p.

Jarosz, Z. and Konopinska, J. 2010. Effect of substrate type and nitrogen fertilization upon yielding and chemical composition of "elsanta" strawberry cultivar grown in unheated foil tunnel. Acta Scientiarum Polonorum, Hortorum Cultus. 9(1):87-96.

Kader, A. A. 1999. Fruit maturity, ripening, and quality relationships. Acta Hortic. 485(1):203208. Doi:10.17660/ACTAHORTIC.1999.485.27.

Konno, H.; Yamaya, T. M.; Yamasaki, Y. and Matsumoto, H. 1984. Pectic polysaccharide breakdown of cell walls in cucumber roots grown with calcium starvation. Plant Physiol. 76(3):633-637. Doi: https://doi.org/10.1104/pp.76.3.633.

Krüger, E.; Josuttis, M.; Nestby, R.; Toldam-andersen, T. B.; Carlen, C. and Mezzetti, B. 2012. Influence of growing conditions at different latitudes of europe on strawberry growth performance, yield and quality. J. Berry Res. 2(3):143-157. Doi: 10.3233/JBR-2012-036.

Li, S. X.; Zhao-Hui, W. and Stewart, B. A. 2003. Responses of crop plants to ammonium and nitrate. N. Adv. Agron. 118(1):205-397. Doi: https://doi.org/10.1016/B978-0-12-4059429.00005-0.

Lieten, P.; Longuesserreet, J.; Baruzzi, G. and Lopez-Medina, J. 2004. Recent situation of strawberry substrate culture in Europe. Acta Hortic. 649(1):193-196. Doi: 10.17660/ActaHortic.2004.649.35.

Marques, G. N. 2016. Sistemas abertos e fechados de cultivo. In.: Rampazzo, E. F. Shimizu, H. K.; Peil, R. M. N.; Fermino, M. H.; Furlani, P. R.; Vidal, H. R. y Zawadneak, M. A. C. and Eneida, M. D. Cultivo de morangueiro em substrato. Curitiba: SENAR-PR. 112 p.

Majerowicz, N.; Kerbauy, G. B.; Nievola, C. C. and Suzuki, R. M. 2000. Growth and nitrogen metabolism of Catasetum fimbriatum (Orchidaceae) grown with different nitrogen sources. Environ. Exp. Bot. 44(3):195-206. Doi: https://doi.org/10.1016/S0098-8472(00)00066-6.

Hawkesford, M.; Horst, W.; Kichey, T.; Lambers, H.; Schjoerring, J.; Moller, I. S. and White, P. 2012. Functions of macronutrients. In: Marschner, P. Marschner's mineral nutrition of higher plants, third (Ed.). UK. Academic press, London. 135-151 p. 
Neri, D.; Baruzzi, G.; Massetani, F. and Faedi, W. 2012. Strawberry production in forced and protected culture in Europe as a response to climate change. Canada. Can. J. Plant Sci. 92(6):1021-1036. Doi: 10.4141/cjps2011-276.

Nestby, R.; Lieten, F.; Pivot, D.; Raynal-Lacroix, C. and Tagliavini, M. 2004. Influence of mineral nutrients on strawberry fruit quality and their accumulation in plant organs: a review. Int. J. Fruit Sci. 5(1):141-158. Doi: https://doi.org/10.1300/J492v05n01_13.

Paranjpe A.; CantliffeI, D. J.; Lamb, E. M.; Stoffella, P. J. and Powell, C. 2003. Winter strawberry production in greenhouses using soilless substrates: an alternative to methyl bromide soil fumigation. Hortic. Sci. Proceedings Florida State. 116(1):98-105.

Nepar. 2019. Núcleo Estadual de sociedade brasileira de ciência do solo. Manual de adubação e calagem para o estado do paraná. Nepar. $2^{\mathrm{a}}$ (Ed.). 289 p.

Othman, Y.; Bataineh, K.; Al-Ajlouni, M.; Alsmairat, N.; Ayad, J.; Shiyab, S.; Qarallah, B. and Hilaire, R. 2019. Soilless culture: management of growing substrate, water, nutrient, salinity, microorganism and product quality. Fresenius Environmental Bulletin. 28(4):3249-3260.

Pires, R. C. M.; Folegatti, M. V. and Passos, F. A. 1999. Estimativa da área foliar de morangueiro. Horticultura Brasileira. 17(2):86-90.

Porra, R. J. 2002. The chequered history of the development and use of simultaneous equations for the accurate determination of chlorophylls a and b. Photosynth. Res. 73(1-3):149-156.

Rampazzo, E. F. 2016. Instalações para cultivo de morangueiro fora do solo. In: Shimizu, H. K.; Marques, G. N.; Peil, R. M. N.; Fermino, M. H.; Furlani, P. R.; Vidal, H. R. and Zawadneak, M. A. C. and Eneida, M. D. Cultivo de morangueiro em substrato. Curitiba: SENAR-PR. $112 \mathrm{p}$.

Recamales, Á. F.; López Medina, J. and Hernanz, D. 2007. Physicochemical characteristics and mineral content of strawberries grown in soil and soilless system. J. Food Quality. 30(5):837-853. Doi: 10.1111/j.1745-4557.2007.00154.x.

Roosta, H. R.; Sajjadinia, A.; Rahimi, A. and Schjoerring, J. K. 2009. Responses of cucumber plant to $\mathrm{NH}_{4}{ }^{+}$and $\mathrm{NO}_{3}{ }^{-}$nutrition: The relative addition rate technique $v s$ cultivation at constant nitrogen concentration. Sci. Hortic. 121(4):397-403. Doi: 10.1016/j.scienta.2009.03.004.

Samec, D.; Maretić, M.; Lugarić, I.; Mešić, A.; Salopek-sondi, B. and Duralija, B. 2016. Assessment of the differences in the physical, chemical and phytochemical properties of four strawberry cultivars using principal component analysis. Food Chem. 194(1):828-834. Doi:10.1016/j.foodchem.2015.08.095.

Silva, F. A. S. and Azevedo, C. A. V. 2002. The Assistat Software Version 7.7 and its use in the analysis of experimental data. Afr. J. Agr. Res. 11(39):3733-3740.

Sokri, S. M.; Babalar, M.; Barker, A. V.; Lesani, H. and Asgari, M. A. 2015. Fruit quality and nitrogen, potassium, and calcium content of apple as influenced by nitrate: Ammonium ratios in tree nutrition. J. Plant Nutr. 38(10):1619-1627.

Tabatabaei, S. J.; Yusefi, M. and Hajiloo, J. 2008. Effects of shading and $\mathrm{NO}_{3}: \mathrm{NH}_{4}$ ratio on the yield, quality and $\mathrm{N}$ metabolism in strawberry. Sci. Hortic. 116(3):264-272. Doi: 10.1016/j.scienta.2007.12.008.

Taiz, L.; Zeiger, E.; Møller, I. M. y Murphy, A. 2017. Fisiologia vegetal e desenvolvimento vegetal. 6 (Ed.). Porto Alegre. 719 p.

Tu, B.; Liu, C.; Tian, B.; Zhang, Q.; Liu, X. and Herbert, S. J. 2017. Reduced abscisic acid content is responsible for enhanced sucrose accumulation by potassium nutrition in vegetable soybean seeds. J. Plant Res. 130(3):551-558. DOI: 10.1007/s10265-017-0912-x. 
Waller, P. L. and Wilson, G. C. S. 1984. Evaluation of growing media for consumer use. Acta Hortic. 150(1):51-58. Doi: 10.17660/ActaHortic.1984.150.5.

Wehr, J. B.; Menzies, N. W. and Blamey, F. P. C. 2004. Inhibition of cell-wall autolysis and pectin degradation by cations. Plant Physiol. Biochem. 42(6):485-492. Doi: https://doi.org/ 10.1016/j.plaphy.2004.05.006.

White, P. J. 2012. Ion uptake mechanisms of individual cells and roots: short-distance transport. In: marschner, P. Marschner's mineral nutrition of higher plants. Third (Ed.). 672 p.

Zhang, W.; Zhang, N. S.; Zhao, J. J.; Guo, Y. P.; Zhao, Z. Y. and Mei, L. X. 2017. Potassium fertilization improves apple fruit (Malus domestica borkh. Cv. Fuji) development by regulating trehalose metabolism. J. Hortic. Sci. Biotechnol. 92(5):1-11. Doi: https://doi.org/10.1080/14620316.2017.1304165.

Zhang, J.; Lv, J.; Dawuda, M. M.; Xie, J.; Yu, J.; LI, J.; Zhang, X.; Tang, C.; Wang, C. and Gan, Y. 2019. Appropriate ammonium-nitrate ratio improves nutrient accumulation and fruit quality in pepper (Capsicum annuum L.). Agronomy. 9(11):683. Doi: https://doi.org/ 10.3390/agronomy9110683. 